\title{
Slow magnetoacoustic-like waves in post-flare loops
}

\author{
A. N. Kryshtal and S. V. Gerasimenko \\ Department of Cosmic Plasma Physics, Main Astronomical Observatory of the National Academy of Sciences of Ukraine, \\ 03680 Zabolotnogo Str., 27, Kiev 127, Ukraine \\ Received 12 February 2003 / Accepted 16 February 2004

\begin{abstract}
We investigate the stability to the development of plasma waves in the preflare situation of a loop structure at the chromospheric part of a current circuit of a loop. We investigate the conditions under which low-frequency plasma instabilities can develop, assuming the absence of beam instabilities. The large-scale quasi-static electric field in the loop circuit is assumed to be "subdreicer" and weak. Thus the percentage of "runaway" electrons is very small and their influence on the process of instability development is negligible. The pair Coulomb collisions are described by a BGK-model integral. We consider the situation when the plasma at the surface layer of a loop has a spatial gradient of density. In accordance with Heyvaerts-Priest-Rust theory, such a preflare situation would typically exist when the amplitude of the weak electric field $\left|\boldsymbol{E}_{0}(t)\right|$ in the circuit of an "old" loop in an active region begins to increase when "new" magnetic flux emerges from under the photosphere. We have found that two types of waves are generated in such a plasma due to the growth of instabilities: the "kinetic Alfven-like" waves and new type of waves, in the range of magnetoacoustic ones. The instability of these latter waves has a clear threshold and it can be considered as an "indicator" of the development of a preflare situation in an active region.
\end{abstract}

Key words. plasmas - Sun: flares - Sun: chromosphere

\section{Introduction}

Plasma instabilities are traditionally assumed to be one of the main sources of waves in the flare atmosphere (Priest 1982; Somov 1994; Zaitsev et al. 1994). Their hierarchy is usually considered as an important element in the theory of cyclotron maser emission (CME) (Melrose 1989; Zaitsev et al. 1994; Mel'nikov et al. 2002) as well as in the dynamics of current sheets which form in flaring arcades (Podgorny \& Podgorny 2001), namely where flares most frequently occur (De Jager 1959; Priest 1982). Thus the loops of arcades are "post-flare" loops of a previous flare and the preflare of the next one. The investigation of the preflare plasma state on the basis of Heyvaerts-Priest-Rust (HPR) theory (Heyvaerts et al. 1977; Podgorny \& Podgorny 2001) is current practice. In this theory a flare is considered to be the result of the interaction of a "new" magnetic flux, which emerges from under the photosphere and an "old" one, which passes through a current circuit of a loop in an arcade. Such interaction results in an adiabatic slow increase of the amplitude of a weak quasi-static electric ("DC") field in the circuit of the "old" loop. From general physical considerations (De Jager 1959; Kadomtsev \& Pogutse 1967) it is clear that, in such circumstances, different low-energetic wave instabilities of the "stream" type will grow in plasma. If the growth time of the large-scale electric field amplitude $\left|\boldsymbol{E}_{0}(t)\right|$ is much larger than the time of instability development, - this

Send offprint requests to: A. N. Kryshtal,

e-mail: kryshtal@mao.kiev.ua situation occurs most frequently (Poletto \& Kopp 1986), then the mechanism of "direct initiation" of instability by the electric field will take place. The corresponding instabilities will have a clearly expressed threshold character (Kryshtal $\&$ Kucherenko 1995). The value of the amplitude $\left|\boldsymbol{E}_{0}(t)\right|$, expressed in units of the local Dreicer field $E_{\mathrm{D}}$, will be the threshold value in this case. We assume that in preflare situation there are only very few "super-energetic" charged particles (i.e. plasma "without beams"). When the electric field is weak, i.e. when

$\varepsilon_{\mathrm{R}} \equiv \frac{E_{0}}{E_{\mathrm{D}}} \ll 1$,

the percentage of "runaway" electrons is so small that their influence on the instability development can be regarded as negligible (Alexandrov et al. 1988). Of course,this is only one of the possible scenarios for the preflare situation development (Zaitsev et al. 1994).

In connection with the "perpetual" problem of solar physics, the problem of coronal heating, Aschwanden (2001) has formulated interesting conclusions based on the recent soft X-ray and EUV data from space observations with the YOHKOH, SOHO and TRACE satellites. We consider that the inclusion of the chromosphere is very important not only "in conventional AC and DC models" (Aschwanden 2001) but in the investigation of the dynamics of the preflare plasma state in a loop in general. Preferential footpoint heating (Aschwanden 2001) can be explained from our point of view in the framework of the well-known Ionson model 
(Ionson 1978). The most important necessary condition is the generation of the kinetic Alfven waves (KAW) on the "chromospheric floor" of the loop current circuit. These waves propagate almost perpendicular to the direction of the magnetic field $\boldsymbol{B}_{0}$ of the loop in its surface layer (Ionson 1978). Second, the observed overdensity of order $q_{n} \approx 10 \div 20$ (Aschwanden 2001), which, of course, is not less in the chromosphere, seems to be a good reason to assume that strong gradients of the plasma density can have a larger effect than the similar values of temperature and magnetic field amplitude $\left|\boldsymbol{B}_{0}\right|$. Here we denote by $\nabla_{L} \equiv \frac{\partial}{\partial x}\left[\ln n_{0 \alpha}(x)\right] \approx \frac{1}{L_{\alpha}}$ the inverse of the gradient scale $L_{\alpha}, \nabla_{L}$ is the reduced spatial gradient of density. The value of $\nabla_{L}$ is proportional to the inverse value of the mean scale of the density inhomogeneity of the electrons and ions $(\alpha=e, i)$. Analogous values for the temperature and the magnetic field can be introduced in a similar way. Since the end of the 1950s (De Jager 1959), it is well known that overdensity is the most distinguishing property of post-flare loops. When the effect of the reduced spatial gradient of density dominates, it is possible to neglect the shear influence on the instability development. This needs some special condition regarding the plasma state and the characteristics of the perturbation. The increasing number of detections of flarelike events at temperatures of $T \approx 1 \div 2 \mathrm{MK}$ in EUV (with SOHO/ETT and TRACE) (Aschwanden 2001) allows us to assume that the early stage of the preflare process can correspond to a temperature of $\sim 0$, $5 \mathrm{MK}$. The plasma parameters should be obtained from the well-known semiempirical model of chromospheric flare regions (Machado et al. 1980).

We investigate the physical conditions for the growth of low-frequency plasma wave instabilities in the case of the longwave perturbations propagating almost perpendicular to the direction of the loop magnetic field $\boldsymbol{B}_{0}$. We use a local rectangular Cartesian coordinate system with the $Z$-axis directed along the field vectors $\boldsymbol{E}_{0}$ and $\boldsymbol{B}_{0}$ (we assume that $\boldsymbol{E}_{0} \| \boldsymbol{B}_{0}$ ). Taking into account that we consider a plasma in the "chromospheric floor" near the footpoint of a loop, this means that the $X Y$-plane is actually parallel to the surface of the photosphere. For intermediate calculations we have used a cylindrical coordinate system in velocity space $\left(v_{\mathrm{II}}=v_{z}, v_{\perp}, \varphi\right)$ (Alexandrov et al. 1988).

We assume that the plasma has a one-dimensional spatial gradient of its density along the $X$-axis. The thickness of the surface layer of the loop is the mean spatial scale of inhomogeneity of the plasma density. For the local solutions of the dispersion relation for the low-frequency plasma waves (Michailovsky 1963) we consider that the origin of the Cartesian coordinates is placed near the inner border of the surface layer. We assume (Michailovsky 1963), that the wave frequency $\omega$ satisfies the inequality

$\omega \ll \Omega_{\mathrm{i}}$

where $\Omega_{\mathrm{i}} \equiv \frac{e B_{0}}{m_{\mathrm{i}} c}$ is the local ion gyrofrequency.

\section{Main physical conditions and approximations for the preflare plasma model}

In the present investigation, we use the same mechanism of "direct initiation" of instability and the same plasma model as in our previous work (Kryshtal \& Kucherenko 1995; Kryshtal 2000), the most important properties of which are the following:

1. The weak large-scale electric field $\boldsymbol{E}_{0}(t)$ is quasi-static, which implies that

$\frac{\partial}{\partial t}\left[\ln \left|\boldsymbol{E}_{0}(t)\right|\right] \ll \tau_{\text {inst }}^{-1} \approx \gamma$.

This can be considered as typical for flares processes and for different types of plasma instabilities (Zaitsev et al. 1994).

On the other hand there is observational evidence for a correlation between the time of maximum $E_{0}(t)$ and the moment at which the first energy release occurs in a flare (Poletto \& Kopp 1986; Zaitsev et al. 1994). The left-hand side of inequality (3) approximately equals the value $T_{\max }^{-1}$, where $T_{\max }$ is the time for the amplitude $E_{0}(t)$ to grow to its maximum value.

We assume that the electric field under consideration is extremely weak and that the threshold value $\left(\varepsilon_{\mathrm{R}}\right)_{\text {bound }}$, at which the instability, with growth rate $\gamma$ and growth time $\tau_{\text {inst }}$, starts developing, does not much exceed the equilibrium value $\left(\varepsilon_{\mathrm{R}}\right)_{\mathrm{EQ}}$. This value corresponds to the origin of the preflare process when interaction between the "old" and "new" magnetic fluxes is absent. In this situation $T_{\max }$ equals the time of the short-period prediction of a flare and the instability appearance can be considered in a sense as a "forerunner" of a flare process.

2. As earlier (Kryshtal 2000) the plasma under consideration is assumed to be fully ionized and collisions are described by a BGK model integral (Alexandrov et al. 1988). The equilibrium velocity distribution function for ions is assumed to be pure Maxwellian, but the same function for the electrons is described by a shifted Maxwellian distribution with electron shift velocity:

$u_{\mathrm{e}}=\frac{e E_{0}}{m_{\mathrm{e}} v_{\mathrm{ei}}}$,

here, $v_{\mathrm{ei}}$ is the ion-electron collision frequency. When a weak ("external") electric field exists and there is an enough strong magnetic field in the plasma, the ion-electron collisions dominate (Alexandrov et al. 1988). The contribution of other mutual collisions of charged particles can be taken into account in a phenomenological way with the help of the factor $\sigma_{\text {ef }}$ (Kryshtal \& Kucherenko 1995).

3. Taking into account the real scales of inhomogeneity of the plasma (electron or ion) densities in the loops (Zaitsev et al. 1994) we consider the long-wave approximation for the perturbations:

$z_{\mathrm{e}}=\frac{k_{\perp}^{2} v_{T \mathrm{e}}^{2}}{\Omega_{\mathrm{e}}^{2}} \ll z_{\mathrm{i}}=\frac{k_{\perp}^{2} v_{T \mathrm{i}}^{2}}{\Omega_{\mathrm{i}}^{2}} \ll 1$

where $z_{\mathrm{e}}$ and $z_{\mathrm{i}}$ are the electron and ion kinetic parameters respectively, $k_{\perp}$ is the transverse component of perturbation wave-vector $\boldsymbol{k}\left(k^{2}=k_{\perp}^{2}+k_{z}^{2} ; k_{\perp}^{2}=k_{x}^{2}+k_{y}^{2}\right), v_{T \mathrm{e}}$ and $v_{T \mathrm{i}}$ are respectively the electron and ion thermal velocities and $\Omega_{\mathrm{e}}$ is the electron gyrofrequency. 
4. The spatial density inhomogeneity is supposedly "weak", which means, according to Michailovsky (1963) that the conditions

$\omega_{\mathrm{e}, \mathrm{i}}^{*} \ll \omega$,

where

$\omega_{\mathrm{e}, \mathrm{i}}^{*} \equiv \frac{k_{y} v_{T \mathrm{e}, \mathrm{i}}^{2}}{\Omega_{\mathrm{e}, \mathrm{i}}} \frac{\partial}{\partial x}\left[\ln n_{0 \mathrm{e}, \mathrm{i}}(x)\right]$,

have to be satisfied by the drift frequencies of electrons and ions, respectively.

5. We have used the condition of quasi-neutrality of plasma

$n_{0 \mathrm{e}}=n_{0 \mathrm{i}}$

for the equilibrium densities of the charged particles. When Eq. (8) is valid for an arbitrary $x$, the analogous equation for the spatial gradients of densities is also valid:

$\frac{\partial n_{0 \mathrm{e}}}{\partial x}=\frac{\partial n_{0 \mathrm{i}}}{\partial x}$.

Equation (9) allows us to make use of the well-known simple connection between the drift frequencies of electrons and ions (Michailovsky 1963; Alexandrov et al. 1988)

$\omega_{\mathrm{e}}^{*}=-t \omega_{\mathrm{i}}^{*}$,

where

$t \equiv \frac{T_{\mathrm{e}}}{T_{\mathrm{i}}}$

In our calculations we have assumed that the density profiles in the surface layer have the form

$n_{0 \alpha}(x)=n_{0} \exp \left[-\frac{x}{L_{\alpha}}\right], \quad(\alpha=\mathrm{e}, \mathrm{i})$

and that

$L_{\mathrm{e}}=L_{\mathrm{i}}=L$

is satisfied. In this case the condition (6) is practically equivalent to the well-known inequality (Michailovsky 1963; Kadomtsev \& Pogutse 1967) for plasma with weak spatial inhomogeneity of density

$\frac{\rho_{\mathrm{i}}}{L} \ll 1$,

where $\rho_{\mathrm{i}} \equiv v_{T i} / \Omega_{\mathrm{i}}$ is the ion gyroradius. Equation (14), as a rule, is easily satisfied in the loops (Zaitsev et al. 1994).

6. Under the mentioned above conditions, it is natural to make in the calculation the local approximation to the dispersion relation (DR), which is justified if (Michailovsky 1963)

$\frac{k_{y}}{k_{\perp}^{2} L} \ll \frac{\mu}{\sqrt{\beta_{\alpha}}}, \quad(\alpha=\mathrm{e}, \mathrm{i})$

where $\mu \equiv \sqrt{\frac{m_{\mathrm{c}}}{m_{\mathrm{i}}}} \cong 2.33 \times 10^{-2}$ (for one-charged ions) and $\beta_{\alpha}$ is the well-known "plasma $\beta$ ":

$\beta_{\alpha}=\frac{8 \pi n_{0} k_{\mathrm{B}} T_{\alpha}}{B_{0}^{2}}, \quad(\alpha=\mathrm{e}, \mathrm{i})$.
The local approximation to the DR allows us to neglect the influence of the boundaries on the process of instability development. Since we consider a "low- $\beta$ " plasma (Michailovsky 1963), then the following inequality has to be satisfied (Krall \& Trivelpiece 1973)

$\mu \ll \beta_{\alpha} \ll 1$.

We assume everywhere that

$\left|k_{x}\right|=\left|k_{y}\right| \equiv k_{\perp} / \sqrt{2}$.

Then the condition (15) for the solutions to be local turns out to be even more stringent than the condition

$\frac{\lambda_{\perp}}{L} \ll 1$

for the transverse wave-length of the perturbation. Inequality (19) is the condition of the approximation of geometrical optics.

7. Taking into account the fact that in a surface layer the main spatial inhomogeneity of density is in the direction perpendicular to the magnetic field $\boldsymbol{B}_{0}$, as well as the fact that the low-frequency waves under consideration are of a quasi-electrostatic type, we assume that the condition

$k_{\mathrm{R}} \equiv \frac{k_{z}}{k_{\perp}} \ll 1$

is satisfied for the components of the perturbation wave-vector (Ionson 1978).

8. We take the "longitudinal" phase velocity of the perturbation to vary in the range

$v_{T \mathrm{i}} \ll \frac{\omega}{k_{z}} \ll v_{T \mathrm{e}}$.

This range is typical of the "Alfven-like" and "drift-like" waves in a plasma (Krall \& Trivelpiece 1973). We have also made use of some additional conditions:

9. In the case of "weak inhomogeneity" we consider a "moderately nonisothermal" plasma with

$1 \leq t \leq 6$.

On the one hand this allows one to neglect the possible influence of ion-acoustic turbulence on the process of instability development. Actually it means that

$\varepsilon_{\mathrm{R}}\left(\equiv \frac{E_{0}}{E_{D}}\right)=\frac{u_{\mathrm{e}}}{v_{T \mathrm{e}}}$.

On the other hand, the relatively low values of $t$ in (22) imply that threshold value $(t)_{\text {bound }}$, at which the instability arises, may be low too. This may be important for the problem of pre-heating of the loop foot-points (Aschwanden 2001).

10. We noted that we neglect in our calculations the reduced spatial gradients of temperature and magnetic field compared to that of density. According to Kadomtsev \& Pogutse, this is equivalent to the neglect of the influence of "shear" (Kadomtsev \& Pogutse 1967). For a characteristic 
scale of the perturbation of order $k_{\perp}^{-1}$, i.e. for $x \approx k_{\perp}^{-1}$, this assumption is correct if the condition for the ion "plasma $\beta$ "

$\beta_{\mathrm{i}} \ll z_{\mathrm{i}} k_{\mathrm{R}}^{2}\left(\beta_{\mathrm{A}} \varepsilon_{\mathrm{R}}\right)^{-2}$

is satisfied. In Eq. (24) $\beta_{\mathrm{A}} \equiv \frac{v_{T \mathrm{e}}}{v_{\mathrm{A}}}$, where $v_{\mathrm{A}}=c \frac{\Omega_{\mathrm{i}}}{\omega_{\mathrm{Pi}}}$ is the Alfven velocity and $\omega_{\mathrm{Pi}}$ is the ion plasma frequency. The analogous condition for the electron "plasma $\beta$ " has the form

$$
\beta_{\mathrm{e}} \varepsilon_{\mathrm{R}} \ll \mu \sqrt{z_{\mathrm{i}} t}\left|k_{\mathrm{R}}\right| .
$$

Taking the conditions (5) and (20) into account, these last two inequalities, (24) and (25), point to the importance of the "right choice" of plasma parameters, i.e. appropriate values of the densities and temperatures of the electrons and ions. With the mentioned above restrictions and conditions, this is not so simple. Semiempirical models of the flare chromosphere (Machado et al. 1980) fortunately provide us with necessary parameters. In our calculations we used the following values:

$n_{0 \mathrm{i}}=n_{0 \mathrm{e}}=6.89 \times 10^{10} \mathrm{~cm}^{-3} ; T_{0 \mathrm{i}}\left(=T_{0 \mathrm{e}}\right)=4.47 \times 10^{5} \mathrm{~K}$

We supposed that in the local thermodynamical equilibrium $T_{\mathrm{e}}=T_{\mathrm{i}}$ before the interaction of the "old" and "new" magnetic fluxes, and $T_{\mathrm{e}}$ can exceed $T_{\mathrm{i}}$ according to Eq. (22) in the case of weak inhomogeneity in the early stage of the preflare process. In the paper of Machado et al. (1980) the values of $n_{0 \mathrm{e}}$ and $T_{\mathrm{e}}$ correspond to the height $h=1459 \mathrm{~km}$ above the photosphere. Strictly speaking the magnetic field amplitude in our model cannot be arbitrary. This is clear in view of the conditions and approximations of the employed model. There is still some freedom in the choice of this parameter (Gopasyuk 1987). We assumed $B_{0}=31,3 \mathrm{G}$. This value is very closed to the value $B_{0}=40 \mathrm{G}$ from Aschwanden (1987).

For such magnetic field amplitudes and with the parameters given in Eq. (26) and extremely small values of $\varepsilon_{R}$, the conditions (24) and (25) can be easily satisfied in the case of a weak inhomogeneity. In the opposite case of a strong inhomogeneity, for extremely small values of $k_{\mathrm{R}}$, when instability can develop on the background of turbulence and the value of $\varepsilon_{\mathrm{R}}$ can sharply increase, the conditions (24) and (25) practically determine an upper limit for $\varepsilon_{\mathrm{R}}$.

In the case of weak inhomogeneity, when the ion-electron collisions dominate, we can estimate for the parameters (26) the equilibrium value $\left(\varepsilon_{\mathrm{R}}\right)_{\mathrm{EQ}}$, which corresponds to the steady state in the loop circuit (without interaction of the "new" and "old" magnetic fluxes). If we assume that the loop under consideration is a "semitorus" with small radius $R_{0}$, it can be shown that

$\left(\varepsilon_{\mathrm{R}}\right)_{\mathrm{EQ}}=\frac{I}{\pi R_{0}^{2} n_{0} e v_{T \mathrm{e}}}$.

For a current in this loop $I=1 \times 10^{12} \mathrm{~A}$ (Gopasyuk 1987) and $R_{0}=10^{8} \mathrm{~cm}$ (Zaitsev et al. 1994), we then obtain

$\left(\varepsilon_{\mathrm{R}}\right)_{\mathrm{EQ}}=1.1 \times 10^{-5}$.

Of course, it seems very problematic to obtain the exact value of $R_{0}$, so the estimate (28) can give only an order of magnetude $\left(\varepsilon_{\mathrm{R}}\right)_{\mathrm{EQ}}$.

\section{Modified dispersion relation and the growth rates of instabilities}

The starting point for obtaining the dispersion relation (DR) for this plasma model is the expression for the scalar dielectric permeability of a hot magnetoactive plasma with spatial inhomogeneity. (Alexandrov et al. 1988).

When the electric field in the low-frequency wave in the inhomogeneous medium can be considered as quasi-potential, the equation for this permeability in the inhomogeneous plasma,

$\varepsilon(\omega, \boldsymbol{k}, x)=0$

plays the same role as the standard DR in homogeneous plasma. In this case, Eq. (29) can be considered as the eikonal equation for electrostatic waves in the zero order of geometrical optics. For the low-frequency waves, which satisfy condition (2), Eq. (29) can be simplified in a standard way (Kryshtal 2000) and reduced to the form

$D(\omega, \boldsymbol{k}, x) \equiv \operatorname{Re} D+i \operatorname{Im} D=\varepsilon_{\mathrm{II}}\left(1-\frac{\omega^{2}}{c^{2} k_{z}^{2}} \varepsilon_{\perp}\right)+\frac{k_{\perp}^{2}}{k_{z}^{2}} \varepsilon_{\perp}=0$,

where $\varepsilon_{\mathrm{II}}$ and $\varepsilon_{\perp}$ are the longitudinal and transverse parts of the dielectric permeability respectively, $c$ is the speed of light in vacuum. This form of DR was first investigated by Michailovsky (1963) in the local approximation at $v_{\mathrm{ei}}=0$, $\varepsilon_{\mathrm{R}}=0$ and $t=1$. In the present plasma model with $v_{\mathrm{ei}} \neq 0$, $\varepsilon_{\mathrm{R}} \neq 0$ and $t>1$ the form of the DR remains the same (Kryshtal 2000), but, if we take into account the existence of subdreicer field and the influence of the collisions, additional terms appear in the expressions of $\varepsilon_{\mathrm{II}}$ and $\varepsilon_{\perp}$. These expressions become much more complicated, with terms that describe the ion thermal motion. But the DR becomes suitable for analysis when $\varepsilon_{\mathrm{R}}$ takes extremely small values. This form of the $\mathrm{DR}$, which is modified by the presence of the external electric field, pair Coulomb collisions and ion thermal motion, is refered to as the modified dispersion relation (MDR). Each root of the equation (Krall \& Trivelpiece 1973)

$\operatorname{Re} D(\omega, \boldsymbol{k}, x)=0$

corresponds to a certain kind of plasma wave, with dispersion law $\omega_{m}=\omega_{m}(\boldsymbol{k}, x)$, where $m$ is a number labeling the root. We consider this plasma wave as the solution of MDR. In the present plasma model, $\omega_{m}$ actually depends not on $x$, but on $L$ (see Eqs. (12) $\div(13)$ ). We determine the growth rate (positive or negative) of the instability from the equation

$\gamma_{m}=-\left.\frac{\operatorname{Im} D(\omega, \boldsymbol{k}, x)}{\frac{\partial}{\partial \omega} \operatorname{Re} D(\omega, \boldsymbol{k}, x)}\right|_{\omega=\omega_{m}}$.

In the framework of our approximations, Eq. (31) can be reduced to the polynomial form

$\sum_{i=0}^{4} P_{\mathrm{i}} \Omega^{\mathrm{i}}=0$

with

$$
\begin{aligned}
P_{0}= & 2 \beta_{\mathrm{A}} \varepsilon_{\mathrm{R}} z_{\mathrm{i}} \Omega^{*}-\beta_{\mathrm{A}}^{2} \varepsilon_{\mathrm{R}}^{2}\left(1+z_{\mathrm{i}} t\right) \\
& -\beta_{\mathrm{A}}^{2} \mu^{2}\left(-1+\frac{\tau_{*}^{2}}{2 \pi \mu^{2}}+\frac{\tau_{*} \beta_{A} \nu \Omega^{*}}{\sqrt{2 \pi} t}\right) ;
\end{aligned}
$$




$$
\begin{aligned}
P_{1}= & 2 \beta_{\mathrm{A}} \varepsilon_{\mathrm{R}}\left(1+z_{\mathrm{i}} t\right)-z_{\mathrm{i}} \Omega^{*}+\tau_{*}\left[2\left(\beta_{\mathrm{A}} \varepsilon_{\mathrm{R}}+\Omega^{*}\right)\right. \\
& \left.+\beta_{\mathrm{A}} \varepsilon_{\mathrm{R}}\left(\beta_{\mathrm{A}} \varepsilon_{\mathrm{R}}+\Omega^{*}\right) \frac{\Omega^{*}}{t}+\beta_{\mathrm{A}}^{2} \varepsilon_{\mathrm{R}} v\left(z_{\mathrm{i}} t+\frac{\Omega^{*}}{2}\right)\right]+\beta_{\mathrm{A}}^{2} \varepsilon_{\mathrm{R}}^{2} \frac{\Omega^{*}}{t} \\
& -\beta_{\mathrm{A}}^{2} \mu^{2}\left[\tau_{*} \beta_{\mathrm{A}} v-\left(1-\frac{\tau_{*}^{2}}{2 \pi \mu^{2}}\right) \frac{\Omega^{*}}{t}\right]
\end{aligned}
$$$$
P_{2}=-1-z_{\mathrm{i}} t+\tau_{*}\left[-1-\frac{\beta_{\mathrm{A}}}{2}\left(z_{\mathrm{i}} t+\Omega^{*}\right)-\left(2 \beta_{\mathrm{A}} \varepsilon_{\mathrm{R}}+\Omega^{*}\right)\right.
$$$$
\left.\times\left(\frac{\Omega^{*}}{t}-\frac{\beta_{\mathrm{A}} \varepsilon_{\mathrm{R}}}{2}\right)-\beta_{\mathrm{A}} \varepsilon_{\mathrm{R}}\left(\beta_{\mathrm{A}} \varepsilon_{\mathrm{R}}+\frac{\Omega^{*}}{2}\right)\right]
$$$$
-\beta_{\mathrm{A}}^{2} \mu^{2}\left(1-\frac{\tau_{*}^{2}}{2 \pi \mu^{2}}\right)-2 \beta_{\mathrm{A}} \varepsilon_{\mathrm{R}}\left(\frac{\Omega^{*}}{t}-\frac{\beta_{\mathrm{A}} \varepsilon_{\mathrm{R}}}{2}\right) ;
$$$$
P_{3}=\frac{\Omega^{*}}{t}-2 \beta_{\mathrm{A}} \varepsilon_{\mathrm{R}}-\tau_{*}\left[2 \beta_{\mathrm{A}} \varepsilon_{\mathrm{R}}+\Omega^{*}\left(1-\frac{1}{t}\right)\right],
$$$$
P_{4}=1+\tau_{*},
$$

where

$\tau_{*}=\sqrt{\frac{2 \pi}{z_{\mathrm{i}}} t} \frac{v \mu}{k_{\mathrm{R}}}, v \equiv \frac{\nu_{\mathrm{ei}}}{\Omega_{\mathrm{i}}} \sigma_{\mathrm{ef}} ; \quad \sigma_{\mathrm{ef}} \in[1 ; 2.5]$.

In the Eq. (33)

$\Omega \equiv \frac{\omega}{k_{z} v_{\mathrm{A}}}$

is the reduced frequency (or reduced "longitudinal" phase velocity). At the same time in the Eqs. (34) $\div(38)$

$\Omega_{\mathrm{e}}^{*}=-t \Omega_{\mathrm{i}}^{*} \equiv \Omega^{*}$,

where

$\Omega_{\alpha}^{*}=\frac{\omega_{\alpha}^{*}}{k_{z} v_{\mathrm{A}}}, \quad(\alpha=\mathrm{e}, \mathrm{i})$

are the reduced drift frequencies of the charged particles. In this paper we only investigated the real roots of the algebraic equation of the fourth order (33), because we want to exclude the possible cases of "aperiodic" instability or "aperiodic" damping (Alexandrov et al. 1988). In numerical simulations, we have found that complex roots of Eq. (33) appear at $\sigma_{\text {ef }}>2,5$. This is why we have taken $\sigma_{\text {ef }}=2,5$ as the upper limit of the interval in (39).

Equation (33) can be solved by the standard Euler method, but for extremely small values of $\varepsilon_{\mathrm{R}}$ (actually as $\varepsilon_{\mathrm{R}} \rightarrow 0$ ) it becomes impossible to obtain the solution in an analytical form. So, we had to make use of the numerical calculations based on exact formulae (Mishina \& Proskuryakov 1962). We have used the roots of the resolvent equation. For all roots to be real and positive, and thus to obtain all four roots of the MDR (33) as real, the condition

$$
\boldsymbol{D} \equiv\left(\frac{Q}{2}\right)^{2}+\left(\frac{R}{3}\right)^{3}<0
$$

should be satisfied for the discriminant $\boldsymbol{D}$ of the resolvent equation (Mishina \& Proskuryakov 1962). Here the following notations have been used:

$R \equiv-\frac{P^{2}}{3}-4 r$

$Q \equiv-q^{2}-\frac{2}{3} P\left(\frac{P^{2}}{9}-4 r\right)$,

where $P, q$ and $r$ are the coefficients of the fourth order equation in the reduced form

$y^{4}-P y^{2}+q y+r=0$.

This equation can be obtained from the initial equation

$a \Omega^{4}+b \Omega^{3}+c \Omega^{2}+\mathrm{d} \Omega+e=0$

through the standard transformation

$y=\Omega+\frac{b}{4 a}$.

The resolvent equation of the third order has the form

$z^{3}+2 P z^{2}+\left(P^{2}-4 r\right) z-q^{2}=0$

Equation (43) imposes the most stringent restrictions on the main plasma characteristics of the employed model. However, these restrictions allow us to consider separately the cases of the "weak" and "strong" inhomogeneities.

The obtained real roots of the MDR (33) have to be substituted into formula (32) to analyse the expressions for the growth rates. Only if

$\gamma_{m}>0 \quad(m=1 \div 4)$

the corresponding waves grow during the linear stage of the process of instability development. The reduced growth rates for the all four roots of the MDR (33) have the following form:

$\Gamma_{m} \equiv \frac{\gamma_{m}}{\omega}=\left.\sqrt{\frac{\pi}{2}} \frac{\left(\Omega-\beta_{\mathrm{A}} \varepsilon_{\mathrm{R}}\right)}{\beta_{\mathrm{A}} \cdot \Omega^{2}} \frac{F_{1}}{F_{2}}\right|_{\omega=\omega_{m}} ;(m=1 \div 4)$,

with

$F_{1}=\sum_{i=0}^{6} C_{i} \Omega^{i}$

$F_{2}=\sum_{i=0}^{4} S_{i} \Omega^{i}$

where

$$
\begin{aligned}
C_{0}= & \beta_{\mathrm{A}}^{3}\left\{\beta_{\mathrm{A}} \frac{\tau_{*}}{\pi}\left[\mu^{2}\left(\frac{\Omega^{*}}{t}+2 \beta_{\mathrm{A}} \varepsilon_{\mathrm{R}}\right)-\varepsilon_{\mathrm{R}}^{2} z_{\mathrm{i}} t\right]-\sqrt{\frac{2}{\pi}} \varepsilon_{\mathrm{R}} v z_{\mathrm{i}} t\right\} \\
C_{1}= & \beta_{\mathrm{A}}^{2}\left\{\sqrt{\frac{8}{\pi}} v\left[\varepsilon_{\mathrm{R}} z_{\mathrm{i}} t-\beta_{\mathrm{A}} \mu^{2}\left(\frac{\Omega^{*}}{2 t}-\beta_{\mathrm{A}} \varepsilon_{\mathrm{R}}\right)\right]\right. \\
& \left.+\beta_{\mathrm{A}} \frac{\tau_{*}}{\pi}\left[\beta_{\mathrm{A}} \mu^{2}\left(\frac{\Omega^{*}}{t^{2}}-2\right)-\varepsilon_{\mathrm{R}}\left\{\pi \Omega^{*}-z_{\mathrm{i}} t(1+\pi)\right\}\right]\right\}
\end{aligned}
$$




$$
\begin{aligned}
C_{2}= & \beta_{\mathrm{A}}\left\{\beta _ { \mathrm { A } } \tau _ { * } \left[\frac{\beta_{\mathrm{A}}}{\pi}\left\{3 \beta_{\mathrm{A}}^{2} \mu^{2} \frac{\Omega^{*}}{t}+\varepsilon_{\mathrm{R}} z_{\mathrm{i}} t\left(\beta_{\mathrm{A}} \varepsilon_{\mathrm{R}}-z_{\mathrm{i}} \Omega^{*}\right)\right\}\right.\right. \\
& \left.+\left(1-\beta_{\mathrm{A}} \varepsilon_{r} m R \frac{\Omega^{*}}{t}\right)\left(z_{\mathrm{i}} t+\Omega^{*}\right)\right]-\varepsilon_{\mathrm{R}}\left(\Omega^{*}+3 \beta_{\mathrm{A}} \varepsilon_{\mathrm{R}}\right) \\
& +\sqrt{\frac{2}{\pi} v}\left[\frac{\beta_{\mathrm{A}}^{2} \tau_{*}^{2}}{2 \pi}-z_{\mathrm{i}} t-\beta_{\mathrm{A}}^{2}\left(\mu^{2}-\varepsilon_{\mathrm{R}}^{2}\right)\right. \\
& \left.\left.+\beta_{\mathrm{A}} \varepsilon_{\mathrm{R}} \tau_{*}\left(\beta_{\mathrm{A}} \varepsilon_{\mathrm{R}}+\frac{\Omega^{*}}{2}\right)\right]\right\} ; \\
C_{3}= & \beta_{\mathrm{A}}^{2} \tau_{*}\left[\left(\frac{\Omega^{*}}{t}-\beta_{\mathrm{A}} \varepsilon_{\mathrm{R}}\right)\left(z_{\mathrm{i}} t+\Omega^{*}\right)+\frac{\beta_{\mathrm{A}}}{\pi}\left(2 \beta_{\mathrm{A}} \mu^{2}-\varepsilon_{\mathrm{R}} z_{\mathrm{i}} t\right)\right] \\
& +3 \beta_{\mathrm{A}} \varepsilon_{\mathrm{R}}+\Omega^{*}+\beta_{\mathrm{A}} \varepsilon_{\mathrm{R}} \frac{\Omega^{*}}{t}\left(3+\Omega^{*}\right) \\
& -\sqrt{\frac{2}{\pi}} \beta_{\mathrm{A}} v\left[2 \beta_{\mathrm{A}} \varepsilon_{\mathrm{R}}+\tau_{*}\left(2 \beta_{\mathrm{A}} \varepsilon_{\mathrm{R}}+\Omega^{*}\right)\right]
\end{aligned}
$$$$
C_{4}=-1+3 \beta_{\mathrm{A}}^{2} \varepsilon_{\mathrm{R}}^{2}+\sqrt{\frac{2}{\pi}} \beta_{\mathrm{A}} v\left(1+\tau_{*}\right)-\frac{\Omega^{* 2}}{t}
$$$$
-\beta_{\mathrm{A}} \varepsilon_{\mathrm{R}} \Omega^{*}\left(\frac{3}{t}-1\right)+\beta_{\mathrm{A}}^{2} \tau_{*}\left(z_{\mathrm{i}} t+\Omega^{*}\right) ;
$$$$
C_{5}=-3 \beta_{\mathrm{A}} \varepsilon_{\mathrm{R}}-\Omega^{*}\left(1-\frac{1}{t}\right)
$$$$
C_{6}=1 ;
$$$$
S_{0}=3 \beta_{\mathrm{A}} \varepsilon_{\mathrm{R}} z_{\mathrm{i}} \Omega^{*}+2 \beta_{\mathrm{A}}^{2} \mu^{2}\left(1-\frac{\tau_{*}^{2}}{2 \pi \mu^{2}}+\frac{\Omega^{* 2}}{t^{2}}\right)
$$$$
+\tau_{*}\left\{\sqrt{\frac{2}{\pi}} \beta_{\mathrm{A}}^{3} v\left[\varepsilon_{\mathrm{R}}^{2}\left(\frac{\Omega^{*}}{2}+z_{\mathrm{i}} t\right)-\mu^{2} \frac{\Omega^{*}}{t}\right]\right.
$$$$
\left.-\beta_{\mathrm{A}} \varepsilon_{\mathrm{R}} \Omega^{*}\left(1-\beta_{\mathrm{A}} \varepsilon_{\mathrm{R}} \frac{\Omega^{*}}{t}\right)\right\} \text {; }
$$$$
S_{1}=-z_{\mathrm{i}} \Omega^{*}-\beta_{\mathrm{A}}^{2} \frac{\Omega^{*}}{t}\left[3 \varepsilon_{\mathrm{R}}^{2}+\mu^{2}\left(1-\frac{\tau_{*}^{2}}{2 \pi \mu^{2}}\right)\right]
$$$$
+\tau_{*}\left\{\Omega^{*}\left[1+\beta_{\mathrm{A}} \varepsilon_{\mathrm{R}}\left\{2 \beta_{\mathrm{A}} \varepsilon_{\mathrm{R}}-\frac{1}{t}\left(3 \beta_{\mathrm{A}} \varepsilon_{\mathrm{R}}+\Omega^{*}\right)\right\}\right]\right.
$$$$
\left.+\sqrt{\frac{2}{\pi}} \beta_{\mathrm{A}} v\left[\beta_{\mathrm{A}} \varepsilon_{\mathrm{R}}\left(z_{\mathrm{i}} t+\frac{3}{2} \Omega^{*}\right)-\beta_{\mathrm{A}}^{2} \mu^{2}\right]\right\} ;
$$$$
S_{2}=3 \beta_{\mathrm{A}} \varepsilon_{\mathrm{R}}\left\{\frac{\Omega^{*}}{t}-2 \beta_{\mathrm{A}} \varepsilon_{\mathrm{R}}-\tau_{*}\left[2 \beta_{\mathrm{A}} \varepsilon_{\mathrm{R}}\right.\right.
$$$$
\left.\left.+\Omega^{*}\left(1-\frac{1}{t}\right)\right]+\sqrt{\frac{\pi}{2}} \nu \Omega^{*}\right\}
$$

$S_{3}=6 \beta_{\mathrm{A}} \varepsilon_{\mathrm{R}}-\frac{\Omega^{*}}{t}+\tau_{*}\left[6 \beta_{\mathrm{A}} \varepsilon_{\mathrm{R}}+\Omega^{*}\left(1-\frac{1}{t}\right)\right]-\sqrt{\frac{\pi}{2}} \nu \Omega^{*}$

$S_{4}=-2\left(1+\tau_{*}\right)$.
Numerical simulation has shown that the requirement of the absence of an imaginary part in the roots of MDR (33) has to be supplemented by the condition

$\Gamma_{m} \ll 1, \quad(m=1,2,3,4)$.

Practically, this means that the use of the linear approximation of perturbation theory is valid. These two conditions (43) and (60) allow us to consider separately the cases of "weak" and "strong" inhomogeneity. The case of weak inhomogeneity corresponds to extremely small values of $\varepsilon_{\mathrm{R}}$ and extremely large possible values of $z_{\mathrm{i}}$ and $k_{\mathrm{R}}$. At the same time the conditions (5) and (20) hold for $z_{\mathrm{i}}$ and $k_{\mathrm{R}}$ respectively, as well as condition (22) for $t$. Specifically, we assumed that the parameters $\varepsilon_{\mathrm{R}}, t, z_{\mathrm{i}}$ and $k_{\mathrm{R}}$ vary in the following ranges:

$1,1 \times 10^{-5} \leq \varepsilon_{\mathrm{R}} \leq 10^{-4}$,

$1 \leq t \leq 6$

$0,1 \leq z_{\mathrm{i}} \leq 0,16$

$0,14 \leq\left|k_{\mathrm{R}}\right| \leq 0,16$.

By analogy with $\left(\varepsilon_{\mathrm{R}}\right)_{\text {bound }}$ we designated the value of $t$ at which the growth rate becomes positive by $t_{\text {bound }}$.

For the set of parameters (26) and $B_{0}=31,3 \mathrm{G}$ we have

$\rho_{i}=20,2 \mathrm{~cm} ; \quad \beta_{A}=10,0 ; \quad v=1,92 \times 10^{-2}$

and

$\sigma_{\mathrm{ef}} v=3,0 \times 10^{-2}$ for $\sigma_{\mathrm{ef}}=1,5$

In the framework of our model, the need for a numerical solution evidently increases. For very narrow intervals of variations of $z_{\mathrm{i}}$ and $k_{\mathrm{R}}$ and constant values $t=t_{\text {bound }}$ and $\varepsilon_{\mathrm{R}}=\left(\varepsilon_{\mathrm{R}}\right)_{\text {bound }}$, corresponding areas on the surfaces of the reduced longitudinal phase velocities $\Omega_{m}=\Omega_{m}\left(z_{\mathrm{i}}, k_{\mathrm{R}}\right), \quad(m=1 \div 4)$ are practically flat. Their local "topology" (i.e. existence of the local extrema) does not play any significant role. In this situation the specific ranges for $z_{\mathrm{i}}$ and $k_{\mathrm{R}}$, where condition (43) is satisfied, as well as specific orientation in "parameter space" of these "locally flat" surfaces, seem much more important, because they allow us to determine, in principle, the dispersion law, i.e. the type of plasma wave. Strictly speaking, this is just an estimate, but this estimate turns out to be reasonably good.

\section{Analysis of stability: The numerical simulation and discussion}

The calculations show that four roots of the MDR (33) can be formally split into two pairs. The roots, which we call " $\Omega_{1}$-" and " $\Omega_{2}$-waves", have in the ranges $(61) \div(64)$ very close (but not the same) values of $\left|\Omega_{1}\right|$ and $\left|\Omega_{2}\right|$, but opposite signs $\left(\Omega_{1}\right.$ is positive and $\Omega_{2}$ is negative). The $\Omega_{1}$-wave can be easily interpreted as the "right" (i.e. with $k_{z}>0$ ) kinetic Alfven wave, modified by the pair collisions and large-scale subdreicer electric field. Unfortunately, we cannot interpret the $\Omega_{2}$-wave in the same way as the inverse kinetic Alfven wave. In the framework of the present model we have used in calculations only 
$k_{\mathrm{R}}$ and $z_{\mathrm{e}, \mathrm{i}}$ as variable parameters to obtain the maximum effect. We do not know the details of the polarization of perturbation, so, we cannot say whether the negative values of $\Omega_{2}$ are the result of incompleteness of our description of perturbation, or whether they demonstrate the well-known "parasitic" effect, when negative frequencies appear in DR (Michailovsky 1963; Alexandrov et al. 1988). For the same reason in the other pair of roots we consider only the $\Omega_{4}$-wave (with positive values) and suppose that only this root corresponds to a "real" wave. It is hard enough to determine exactly the dispersion law for this wave. We can only approximately describe it as a wave from the range of the slow magneto-acoustic ones, because in our model the dispersion law for these SMA-waves has the approximate form $\Omega \approx \mu \beta_{\mathrm{A}}$. This wave is definitely modified by the weak drift motions, pair Coulomb collisions and subdreicer electric field. It practically does not depend on $k_{\mathrm{R}}$ (more exactly, this dependence is very weak) and depends on $z_{\mathrm{i}}$ in an unusual way in comparison to KAW. This last fact does not permit us to consider this $\Omega_{4}$-wave as the "exact" SMA-wave, even taking into account the corrections to the dispersion law due to the collisions, subdreicer field and drift motions. Here we meet an interesting phenomenon: at $v_{\mathrm{ei}}=0, t=1$ and $\varepsilon_{\mathrm{R}}=0$ ("Michailovsky's"-case, 1963), without the collisions and electric field, the DR (33) becomes a polynomial of third order. The roots of this DR are the two Alfven (in our case - the kinetic Alfven) waves and slow drift-Alfven wave (which contains in its dispersion law the drift frequency $(41) \div(42)$ as the factor, and because of this is very slow) (Michailovsky 1963). When $v_{\mathrm{ei}} \neq 0, t>1$ and $\varepsilon_{\mathrm{R}} \neq 0$, the KAWs are, of course, modified, but remain KAWs. At the same time the slow drift Alfven wave vanishes, and instead of this the $\Omega_{3,4}$-waves appear, which contain the drift frequency $(41) \div(42)$ in their dispersion laws not as the small factor, but as the small correction. Figures 1 and 2 show the behaviour of the functions $\Omega_{1}=\Omega_{1}\left(z_{\mathrm{i}}, k_{\mathrm{R}}\right)$ and $\Omega_{4}=\Omega_{4}\left(z_{\mathrm{i}}, k_{\mathrm{R}}\right)$ at

$t=t_{\text {bound }}=1.7$;

$\varepsilon_{\mathrm{R}}=\left(\varepsilon_{\mathrm{R}}\right)_{\text {bound }}=6.5 \times 10^{-5}$.

Figures 3 and 4 show the behaviour of the corresponding reduced growth rates $\Gamma_{1} \equiv \Gamma\left(\Omega_{1}\right)=\Gamma_{1}\left(z_{\mathrm{i}}, k_{\mathrm{R}}\right)$ and $\Gamma_{4} \equiv$ $\Gamma\left(\Omega_{4}\right)=\Gamma_{4}\left(z_{\mathrm{i}}, k_{\mathrm{R}}\right)$ at the same values of $t_{\text {bound }}$ and $\left(\varepsilon_{\mathrm{R}}\right)_{\text {bound }}$ as in $(67) \div(68)$. It can be easily seen that $\Gamma_{1}<0$, thus the $\Omega_{1}$-wave (modified KAW) damps. At the same time $\Gamma_{4}$ becomes positive for some values of $z_{\mathrm{i}}$ and $k_{\mathrm{R}}$, when $t \geq t_{\text {bound }}$ and $\left(\varepsilon_{\mathrm{R}}\right) \geq\left(\varepsilon_{\mathrm{R}}\right)_{\text {bound }}$. This means that the instability of the $\Omega_{4}$-wave has a clearly expressed threshold and can be considered as being an indicator of the dynamics of preflare process. In a sense the generation of the $\Omega_{4}$-wave during the linear stage of the process of instability development can be considered as a forerunner of a flare in a loop structure.

An interesting situation occurs for $k_{z}<0$. The $\Omega_{2}$ and $\Omega_{3}$-waves become the physical meaningful ones, and $\Omega_{1,4}$-waves in a sense lose their physical meaning. Figures 5 and 6 show the behaviour of the inverse KAW-like $\Omega_{2}$-wave and the inverse SMAW-like $\Omega_{3}$-wave.Their growth rates $\Gamma_{2}$ and $\Gamma_{3}$ are shown in Figs. 7 and 8 . In this case the $\Omega_{2}$-wave

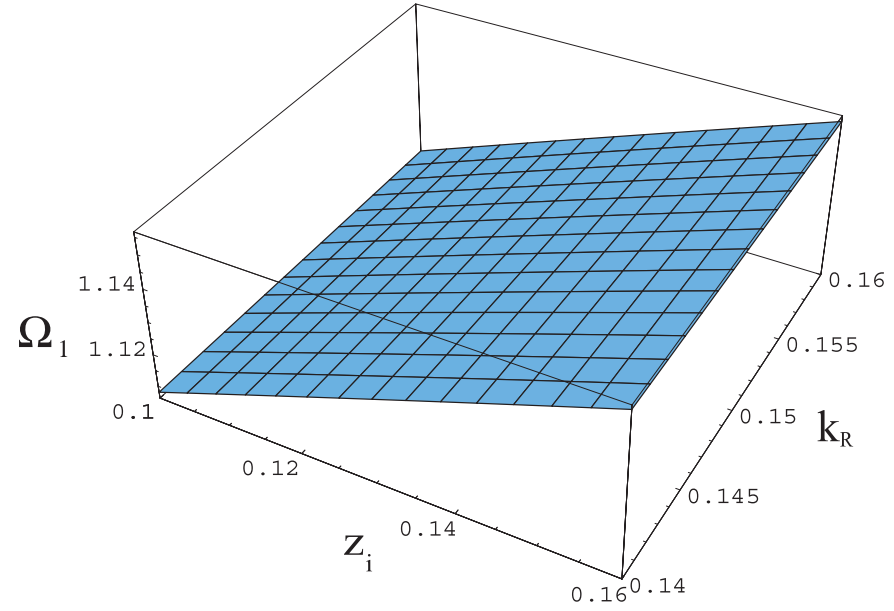

Fig. 1. The KAW-like root of MDR $\Omega=\Omega_{1}=\Omega_{1}\left(z_{\mathrm{i}}, k_{\mathrm{R}}\right)$ at $t_{\text {bound }}=1.7$ and $\left(\varepsilon_{\mathrm{R}}\right)_{\text {bound }}=6.5 \times 10^{-5}$.

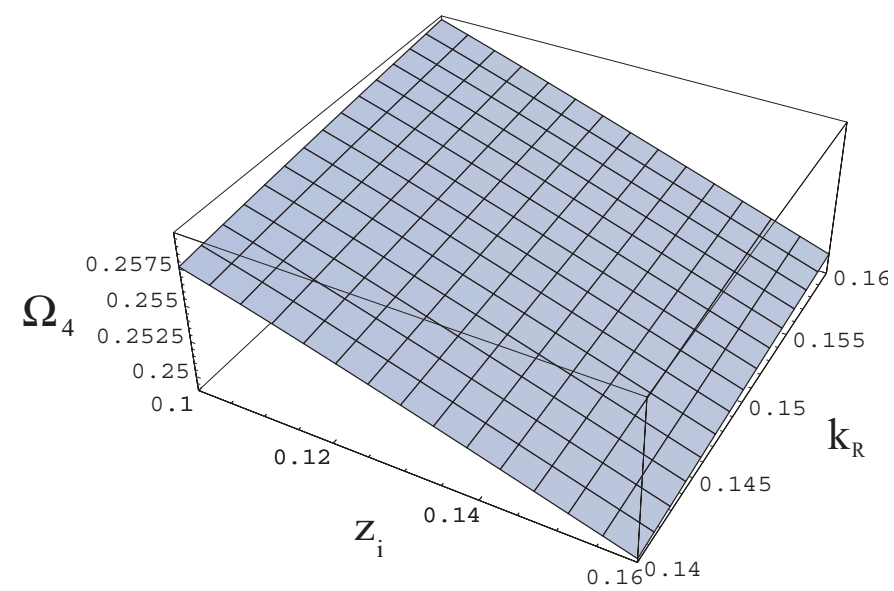

Fig. 2. The SMAW-like root of MDR $\Omega=\Omega_{4}=\Omega_{4}\left(z_{\mathrm{i}}, k_{\mathrm{R}}\right)$ at $t_{\text {bound }}=$ 1.7 and $\left(\varepsilon_{\mathrm{R}}\right)_{\text {bound }}=6.5 \times 10^{-5}$.

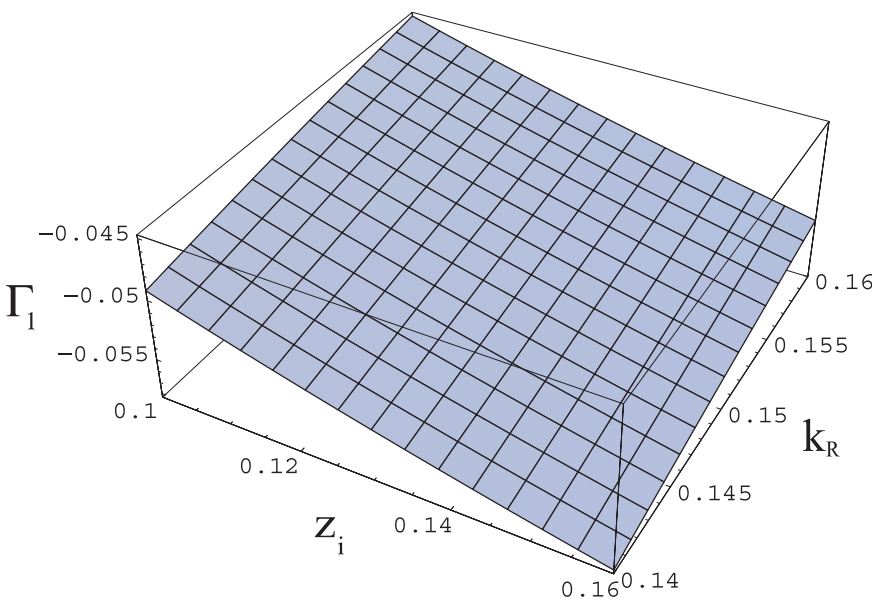

Fig. 3. The growth rate of KAW-like instability $\Gamma_{1}=\Gamma\left(\Omega_{1}\right)=$ $\Gamma_{1}\left(z_{\mathrm{i}}, k_{\mathrm{R}}\right)$ at $t_{\text {bound }}=1.7$ and $\left(\varepsilon_{\mathrm{R}}\right)_{\text {bound }}=6.5 \times 10^{-5}$.

plays the role of a forerunner of a flare. But it has one very important defect: at the same value of $\varepsilon_{\mathrm{R}}=\left(\varepsilon_{\mathrm{R}}\right)_{\text {bound }}=6.5 \times 10^{-5}$ it has too high value of $t_{\text {bound }}=3.3$. In the framework of our model this is important. 


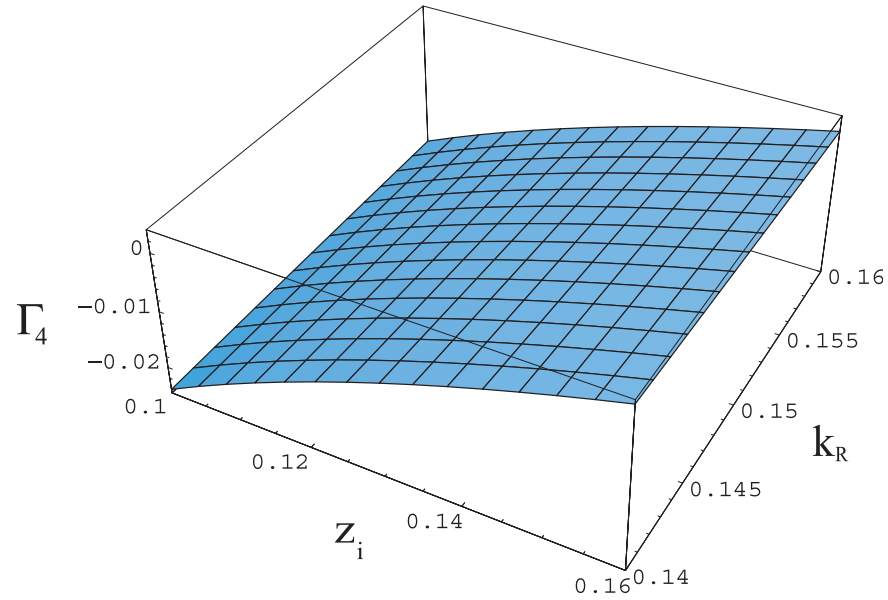

Fig. 4. The growth rate of SMAW-like instability $\Gamma_{4}=\Gamma\left(\Omega_{4}\right)=$ $\Gamma_{4}\left(z_{\mathrm{i}}, k_{\mathrm{R}}\right)$ at $t_{\mathrm{bound}}=1.7$ and $\left(\varepsilon_{\mathrm{R}}\right)_{\mathrm{bound}}=6.5 \times 10^{-5}$.

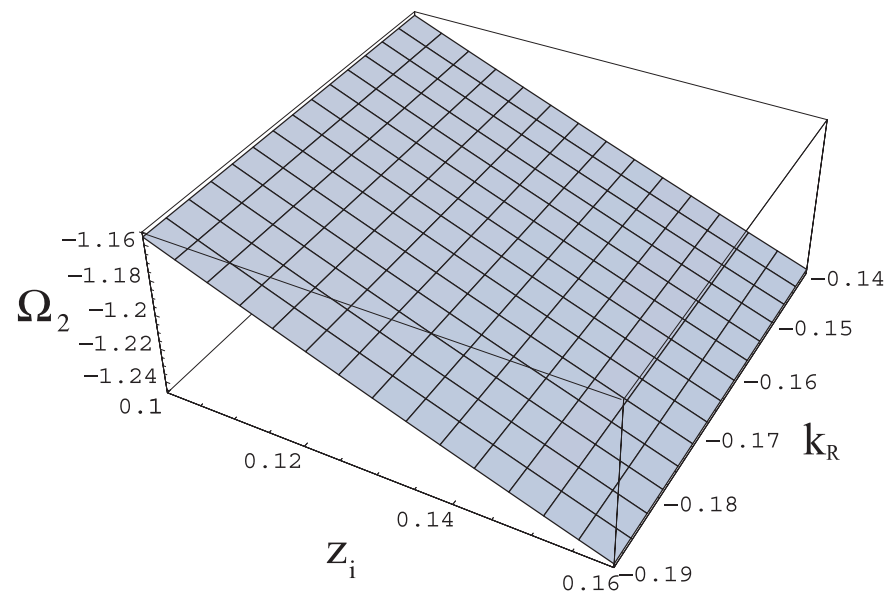

Fig. 5. The inverse KAW-like root of MDR $\Omega=\Omega_{2}=\Omega_{2}\left(z_{\mathrm{i}}, k_{\mathrm{R}}\right)$ at $t_{\text {bound }}=3.3$ and $\left(\varepsilon_{\mathrm{R}}\right)_{\text {bound }}=6.5 \times 10^{-5}$.

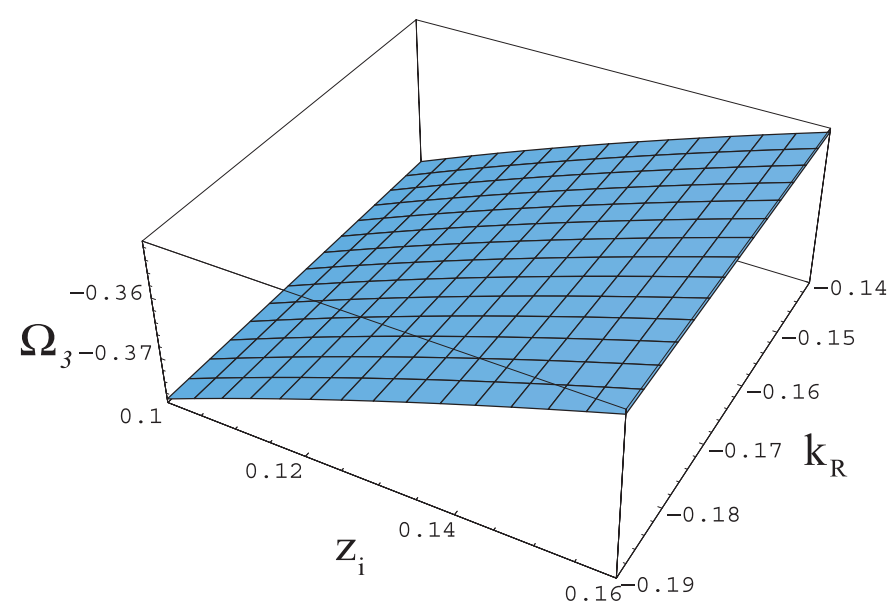

Fig. 6. The inverse SMAW-like root of MDR $\Omega=\Omega_{3}=\Omega_{3}\left(z_{\mathrm{i}}, k_{\mathrm{R}}\right)$ at $t_{\text {bound }}=3.3$ and $\left(\varepsilon_{\mathrm{R}}\right)_{\text {bound }}=6.5 \times 10^{-5}$.

The role of magneto-acoustic waves in the flare models (Priest 1982; Somov 1994; Zaitsev et al. 1994; Zaitsev \& Stepanov 1982), especially in the laboratory modelling of this phenomenon (Somov 1994; Zaitsev et al. 1994), is well-known. There is a considerable number of investigations

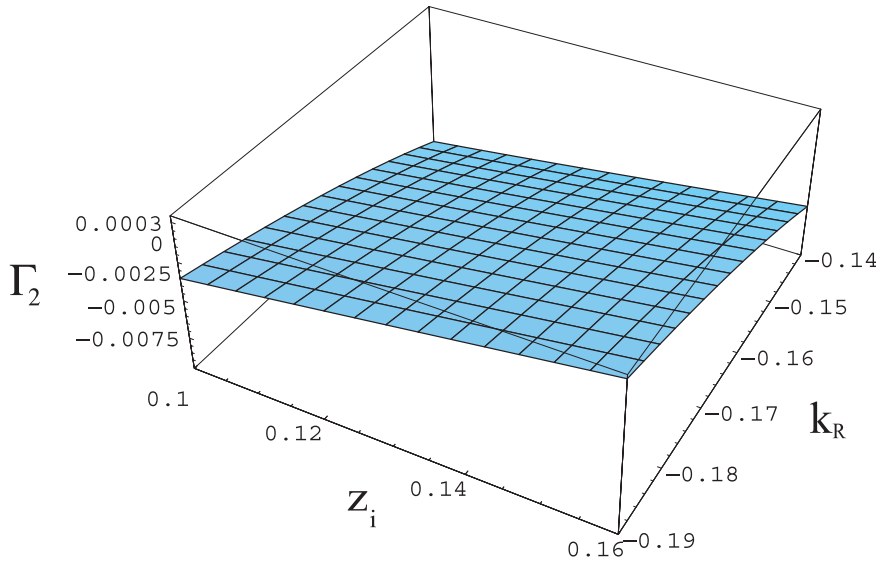

Fig. 7. The growth rate of the inverse KAW-like instability $\Gamma_{2}=$ $\Gamma\left(\Omega_{2}\right)=\Gamma_{2}\left(z_{\mathrm{i}}, k_{\mathrm{R}}\right)$ at $t_{\text {bound }}=3.3$ and $\left(\varepsilon_{\mathrm{R}}\right)_{\text {bound }}=6.5 \times 10^{-5}$.

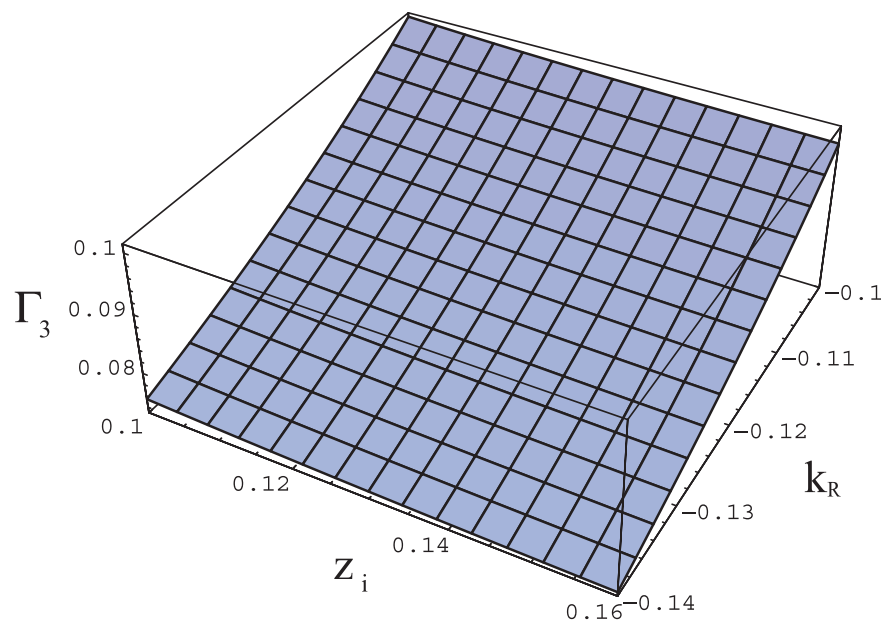

Fig. 8. The growth rate of inverse SMAW-like instability $\Gamma_{3}=$ $\Gamma\left(\Omega_{3}\right)=\Gamma_{3}\left(z_{\mathrm{i}}, k_{\mathrm{R}}\right)$ at $t_{\text {bound }}=3.3$ and $\left(\varepsilon_{\mathrm{R}}\right)_{\text {bound }}=6.5 \times 10^{-5}$.

(Rosenraukh \& Stepanov 1988; Terekhov et al. 2002; Zaitsev \& Stepanov 1989) of the pulsations of the flare emission as well as investigations of the transverse waves in the loop structures (Podgorny \& Podgorny 2001; Mel'nikov et al. 2002; Schrijver et al. 2002). From our point of view, the most interesting peculiarities of the present plasma model and of the results that we have obtained are the following:

1. The instability is of a "non-beam" type. The only analogue of any kind of a beam in this plasma model is the "beam" of "runaway" electrons. Due to the extremely small values of $\varepsilon_{\mathrm{R}}$ in the case of weak inhomogeneity its influence on the process of instability development is negligible.

2. The extremely small value of $\left(\varepsilon_{R}\right)_{\text {bound }}$ from (68) points to the fact that we really study a very early stage of the preflare process (see $\left(\varepsilon_{\mathrm{R}}\right)_{\mathrm{EQ}}$ from $(28)$ ).

3. The relatively small value of $t_{\text {bound }}$ from (67) demonstrates that in the framework of this plasma model no considerable preheating near the foot-points is needed for the generation of an $\Omega_{4}$-wave.

4. The small values of the reduced growth rate $\Gamma_{4}$ definitely point to the fact that we study a clearly expressed wave 
process, with a large number of periods of the generated waves.

Evidently, the generation of an $\Omega_{4}$-wave is not enough for the real short-time prediction of a flare in a loop structure. But it is one of its most important necessary conditions.

Acknowledgements. The authors thank Dr. K. V. Alikajeva and Prof. S. I. Gopasyuk for useful discussions of the present work.

\section{References}

Alexandrov, A. F., Bogdankevich, L. S., \& Rukhadze, A. A. 1988, Principles of Plasma Electrodynamics, Higher School, Moscow, Vys'shaya shkola

Aschwanden, M. I. 1987, Sol. Phys., 111, 113

Aschwanden, M. I. 2001, ApJ, 560, 1035

De Jager, C. 1959, Structure and Dynamics of the Solar Atmosphere (Springer)

Gopasyuk, S. I. 1987, Res. Sci. Tech., Ser. Astron. (USSR), 34, 6

Heyvaerts, I., Priest, E. R., \& Rust, D. 1977, ApJ, 216, 213

Ionson, J. A. 1978, ApJ, 226, 650

Kadomtsev, B. B., \& Pogurse, O. I. 1967, in Problems of Plasma Theory (USSR), ed. M. A. Leontovich, 5, 209

Krall, N. A., \& Trivelpiece, A. W. 1973, Principles of plasma Physics (McGraw-Hill)
Kryshtal, A. N., \& Kucherenko, V. P. 1995, J. Plasma Phys., 53, 169

Kryshtal, A. N. 2000, Kinemat. Phys. Celest. Bod., 16(6), 526

Machado, M. E., Avrett, E. H., Vernazza, J. E., \& Noyes, R. W. 1980, ApJ, 242, 236

Mel'nikov, V. F., Fleishman, G. D., Fu, K. D., \& Huang, G.-L. 2002, Sov. Astron. - AJ, 79(6), 551

Melrose, D. B. 1989, Sol. Phys., 119, 143

Michailovsky, A. B. 1963, in Problems of Plasma Theory (USSR), ed. M. A. Leontovich, 3, 141

Mishina, A. P., \& Proskuriakov, I. V. 1962, Higher Algebra, MPPML, Moscow (in Russian)

Podgorny, A. I., \& Podgorny, I. M. 2001, Sov. Astron. - AJ, 78(1), 71

Poletto, G., \& Kopp, R. A. 1986, in The Lower Atmosphere of Solar Flares, Sacramento Peak, ed. D. F. Neideg, 453

Priest, E. R. 1982, Solar Magnetohydrodynamics (D. Reidel Publ.Co.)

Somov, B. V. 1994, Fundamentals of Cosmic Electrodynamics (Kluwer Acad. Publ.)

Schrijver, C. I., Ashwanden, M. I., \& Title, A. M. 2002, Sol. Phys., 206, 69

Rosenraukh, Yu. M., \& Stepanov, A. V. 1988, Sov. Astron. - AJ, 65, 300

Terekhov, O. V., Shevchenko, A. V., Kuz'min, A. G., et al. 2002, Sov. Astron. Lett., 28, 6, 452

Zaitsen, V. V., \& Stepanov, A. V. 1982, Sov. Astron. Lett., 8, 4, 248

Zaitsen, V. V., \& Stepanov, A. V. 1989, Sov. Astron. Lett., 15, 2, 154

Zaitsen, V. V., Stepanov, A. V., \& Tsap, Yu. T. 1994, Kinemat. Phys. Celest. Bod., 10(6), 3 